\title{
Cytoplasmic translocation of nuclear LSD1 (KDM1A) in human hepatoma cells is induced by its inhibitors
}

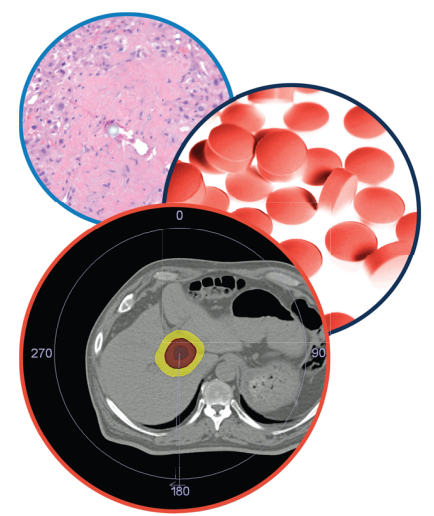

Hepatic Oncology

Suemi Yabuta ${ }^{1} \&$ Yoshihiro Shidoji*,1

${ }^{1}$ Molecular \& Cellular Biology, Graduate School of Human Health Science, University of Nagasaki, 1-1-1 Academy Hills, Nagayo, Nagasaki 851-2195, Japan

*Author for correspondence: shidoj@@un.ac.jp

\begin{abstract}
Aim: Histone-modifiable lysine-specific demethylase-1 (LSD1/KDM1A) is an oncoprotein upregulated in cancers, including hepatoma. We previously reported that the hepatoma-preventive geranylgeranoic acid (GGA) inhibits KDM1A at the same IC $\mathrm{C}_{50}$ as that of the clinically used tranylcypromine. Here, we report that these inhibitors induce the cytoplasmic translocation of nuclear KDM1A in a human hepatoma-derived cell line. Methods \& results: Immunofluorescence studies revealed that KDM1A was cytoplasmically localized in HuH-7 cells $3 \mathrm{~h}$ after GGA or tranylcypromine addition. However, GGA did not affect the subcellular localization of another histone lysine-specific demethylase, KDM5A. This suggests that GGA-induced translocation is KDM1A specific. Conclusion: These data demonstrate, for the first time, that KDM1A inhibitors specifically induce the cytoplasmic translocation of nuclear KDM1A.
\end{abstract}

First draft submitted: 28 December 2018; Accepted for publication: 8 April 2019; Published online: 5 June 2019

Keywords: cytoplasmic translocation • geranylgeranoic acid • hepatoma • LSD1/KDM1A • phospho-histone H3 • tranylcypromine

Epigenetic alterations often promote or drive cancer development by activating and sustaining cancer-promoting gene expression [1]. Among epigenetic changes in cancer cells, histone lysine methylation/demethylation has gained substantial attention as a possible target in therapeutic drug development [2]. Histone lysine methylation/demethylation is extensively involved in nucleosome remodeling and gene expression. Lysine residue methylation is reversibly regulated by histone lysine methyltransferases and histone lysine demethylases (KDMs). Several KDMs have been identified, and KDM1A(LSD1) has received increasing attention since its identification in 2004 [3]. Among enzymes involved in histone modifications, KDM1A is particularly interesting. Overexpression of KDM1A has been reported in acute myeloid leukemia and inhibition of KDM1A activity induces growth suppression and differentiation of retinoid-treated tumor cells [4]. Overexpression of KDM1A is frequently observed in prostate, breast and bladder cancers, neuroblastoma, and in hepatoma [5]. In hepatoma, KDM1A expression directly correlates with adverse clinical outcome and inversely correlates with differentiation [6]. Thus, KDM1A inhibitors are of clinical interest for their anticancer roles as well as their potential application in human diseases with deregulated gene expression [7].

KDM5A/JARID1A, another member of the KDM family, removes a methyl group from the trimethylated lysine 4 of histone $\mathrm{H} 3$ to produce dimethylated lysine 4 . This produces a substrate for KDM1A, which is cooperatively capable of removing dimethyl and monomethyl groups from lysine 4 of histone H3 [7,8]. KDM1A and KDM5A are recruited to chromatin through the CoREST [7] and ORC2-SUMO2 [9] complexes, respectively. Hence, these KDMs are generally recognized as nuclear-localized proteins [10,11].

KDM1A is a flavin adenine dinucleotide-containing enzyme belonging to the amine oxidase superfamily [12]. Structural homology between KDM1A and MAOB, a clinically validated pharmacological target, suggests that KDM1A is a suitable target for drug therapy. Indeed, screening of known monoamine oxidase inhibitors has uncovered KDM1A inhibitors that are effective at submillimolar concentrations. Among these, the best known is 
the clinically used antidepressant drug trans-2-phenylcyclopropylamine (TCP). Multiple clinical trials have begun to investigate the antitumor effect of TCP in acute myeloid leukemia [13].

Recently, we reported that all-trans geranylgeranoic acid (GGA), a natural acyclic diterpenoid found in medicinal herbs [14], inhibits KDM1A activity at the same $\mathrm{IC}_{50}$ as that of TCP [15]. This observation was further validated using the novel TOF mass spectrometry method [16]. GGA induces cell death in several human hepatoma cell lines [14,17]. Incomplete autophagic response [18], nuclear translocation of cytoplasmic p53 [19] and endoplasmic reticulum stress response [20] have been explored as potential mechanisms of GGA-induced cell death. Indeed, the 4,5-didehydro derivative of GGA potentially prevents second primary hepatoma in placebo-controlled randomized Phase II-III clinical trials [21-24].

To our knowledge, no studies have reported KDM1A inhibitor-induced changes in the subcellular distribution of the enzyme. Studying the growth-suppressing effects of GGA in hepatoma cells we discovered that GGA altered the subcellular localization of KDM1A. This could be important for both KDM1A-inhibitor screening and understanding KDM1A biology. Here, we report that treatment with some KDM1A inhibitors results in the removal of KDM1A from chromatin and relocation to the cytoplasmic space in human hepatoma cells.

\section{Materials \& methods}

Materials

GGA and farnesoic acid (FA) were generous gifts from Kuraray (Okayama, Japan). Geranylgeraniol (GGOH) and TCP hydrochloride were purchased from Sigma Aldrich (MO, USA). All-trans retinoic acid (ATRA) was obtained from Wako Pure Chemicals (Osaka, Japan).

\section{Cell culture \& treatment}

Human hepatoma-derived HuH-7 cells were seeded at $1.5 \times 10^{4}$ cells $/ \mathrm{cm}^{3}$ and cultured in Dulbecco's modified Eagle medium ( $4500 \mathrm{mg} / \mathrm{l}$ glucose, Wako Pure Chemicals) containing 5\% heat-inactivated fetal bovine serum (Thermo Scientific Hyclone, Yokohama, Japan) for 2 days. Thereafter, the medium was replaced with fetal bovine serum-free Dulbecco's modified Eagle medium 1 day before the introduction of relevant test compounds at the required concentration. Ethanol $(0.1 \%, \mathrm{v} / \mathrm{v})$ was used as a vehicle control.

\section{Immunofluorescence}

After drug treatment, cells cultured on glass inserts in a 24 -well plate were rinsed with $\mathrm{Ca}^{2+}$-free Dulbecco's phosphate buffered saline (PBS [-], Sigma Aldrich), and the cells were incubated at $4^{\circ} \mathrm{C}$ overnight with appropriate primary antibodies. The primary antibodies used were rabbit polyclonal anti-LSD1 (or KDM1A; Cell Signaling Technology, MA, USA) and rabbit monoclonal anti-JARID1A antibody (KDM5A; D28B10, Cell Signaling Technology). After being washed with PBS [-]), the cells were incubated at room temperature for $1 \mathrm{~h}$ with Alexa Fluor 488-labeled goat anti-rabbit IgG antibody (Invitrogen, Molecular Probes, Tokyo, Japan) and/or antiphospho-histone H3 (Ser10; pHH3)-Alexa Fluor ${ }^{\circledR} 647$ conjugate (Cell Signaling Technology) and the nuclei were counter-stained with Hoechst 33258 (Invitrogen). After being washed with PBS [-], the cells were mounted in PermaFluor (Beckman Coulter, CA, USA), covered with a glass slide, and observed under an LSM700 2Ch Laser module URGB (pigtailed; 405[U], 488[B], 561 [G], 640[R] nm) confocal laser-scanning fluorescence microscope equipped with an Axio Observer Z1 Bio (Carl Zeiss, Göttingen, Germany). Microscopic images were acquired and quantitatively analyzed using Zen 2010B SP1 software (LSM700 version 6.0, Carl Zeiss). A Z-stack procedure was employed, and the acquired images were processed with Zen 2010B SP1, and 3D rendering was performed with Imaris x64 version 7.1.0 (Bitplane Scientific Software, Zurich, Switzerland).

\section{Cell-free analysis of drug-induced release of nuclear KDM1A}

HuH-7 cell nuclear fractions were prepared for cell-free analysis of inhibitor-induced nuclear KDM1A translocation using a CelLytic ${ }^{\mathrm{TM}}$ nuclear kit (Sigma Aldrich). The nuclear pellets were gently suspended in PBS-T (PBS containing $0.1 \%$ Triton-X 100), then GGA or TCP (final concentrations 0-20 $\mu \mathrm{M}$ ) was added to each nuclear suspension (60 $\mu \mathrm{g}$ protein/tube). Proteins were quantified by Bradford assay (Bio-Rad, CA, USA), and samples were gently vortexed and incubated overnight at $4{ }^{\circ} \mathrm{C}$. The supernatants were centrifuged at $1000 \times \mathrm{g}$ for $10 \mathrm{~min}$, separated by SDS-PAGE, and analyzed by western blotting with an anti-KDM1A antibody as described below. 


\section{Western blotting}

The eluted proteins in equal volume were separated by SDS-PAGE and transferred to semi-dry blotted polyvinylidene fluoride membranes (Bio-Rad). The membranes were probed with a rabbit polyclonal antibody against KDM1A (Cell Signaling Technology) or a rabbit polyclonal anti-lamin A antibody (Sigma Aldrich) to stain loading control, and then with horseradish peroxidase (HRP)-labeled secondary antibody (GE Healthcare, Tokyo, Japan). KDM1A bands were detected with Immobilon Western Chemiluminescent HRP substrate (Merck Millipore, Japan) using Image Quant LAS 4000 (GE Healthcare).

\section{Reverse transcription real-time (quantitative) PCR (RT-qPCR)}

Total RNA was isolated using the High Pure RNA Isolation Kit (Roche Diagnostics, Basel, Switzerland). cDNA was generated using the Transcriptor First Strand cDNA Synthesis Kit with random hexameters (Roche). PCR primers used were: KDM1A Fw, CCAGGTGCCCCACAGCCGAT; KDM1A Rv, GCCTGGCGAGGCAGCGTATA; $28 S$ rRNA Fw, TTAGTACGCGCATGAATGG; and $28 S$ rRNA Rv, TGTGGTTTCGCTGGATAGTAGGT. RTqPCR of the cDNA preparations (100 ng each) was performed with Roche Faststart DNA Master SYBR Green I (Roche) on a LightCycler1.5 or 96 (Roche), following manufacturer's instructions.

\section{Statistical analysis}

Differences between two groups were assessed by Student's t-test and were considered statistically significant if the p-value was $<0.05$. All experiments described in this study were performed at least in triplicate.

\section{Results}

\section{Cytoplasmic translocation of nuclear KDM1A induced by GGA or TCP}

Nuclear localization of the KDM1A chromatin protein was confirmed in control cells by immunofluorescence. A strict colocalization of KDM1A with Hoechst 33258-stained chromatin DNA was observed in control cells (Figure 1A). However, in HuH-7 cells $3 \mathrm{~h}$ after treatment with the KDM1A inhibitors GGA or TCP, the subcellular localization of KDM1A appeared to have shifted from the nucleus to the cytoplasmic space (Figure 1A). Quantitative analysis of KDM1A green fluorescence intensity clearly shows that both inhibitors significantly affected the KDM1A subcellular localization (Figure 1B). This indicates that KDM1A nuclear exclusion was induced by both KDM1A inhibitors.

The exclusion of KDM1A from the nucleus by treatment with its inhibitors was further confirmed by Z-axis projections (top and right parts of each image in the left column of Figure 1C). Z-stack 3D rendering of the three images clearly illustrates that the KDM1A protein was localized outside the nuclei $3 \mathrm{~h}$ after treatment with GGA or TCP, whereas most KDM1A was sequestered in the nuclei of the control cells (the right column of Figure 1C).

Nair et al. have reported that KDM1A is recruited to the chromatin of rodent embryonic stem cells in G1/S/G2 phases and is displaced from the chromatin of $\mathrm{M}$-phase cells [25]. Therefore, we next examined whether this was also the case in human hepatoma-derived HuH-7 cells. In M-phase cells stained with anti-pHH3, KDM1A protein was distributed throughout the inside of the cell. In another two cells, not in M-phase and to the right of the M-phase cell, the protein was retained in the nucleus (the upper row of images in Figure 1D). In cells treated with a KDM1A inhibitor (GGA or TCP), KDM1A was distributed throughout the whole cell or outside the nucleus in all cells irrespective of cell-cycle stage (images in lower two tiers of Figure 1D).

\section{Specificity for KDM1A inhibitors \& nuclear KDMs}

Having identified GGA-induced translocation of nuclear KDM1A for the first time, we next considered whether this cytoplasmic translocation could be induced by treatment with a similar class of molecule. We specifically wanted to assess the effects of the diterpenoid of ATRA that lacks KDM1A-inhibitory activity, or of weak inhibitor isoprenoids of GGOH and FA [15]. ATRA did not induce cytoplasmic translocation of nuclear KDM1A. GGOH and FA less efficiently, but significantly, induced cytoplasmic release of nuclear KDM1A (Figure 2A), suggesting that the translocation response of nuclear KDM1A is associated with its inhibition.

Next, we tested whether GGA or TCP could induce cytoplasmic translocation of another nuclear KDM, KDM5A, which acts cooperatively with KDM1A to remove methyl groups from histone H3 lysine 4. KDM5A was localized only in the nuclei of control cells (Figure 2B). Neither GGA nor TCP, inhibitors of KDM1A but not of KDM5A, induced cytoplasmic translocation of nuclear KDM5A. This observation was further confirmed by $\mathrm{Z}$-axis projections (top and right parts of each image in Figure 2C). 


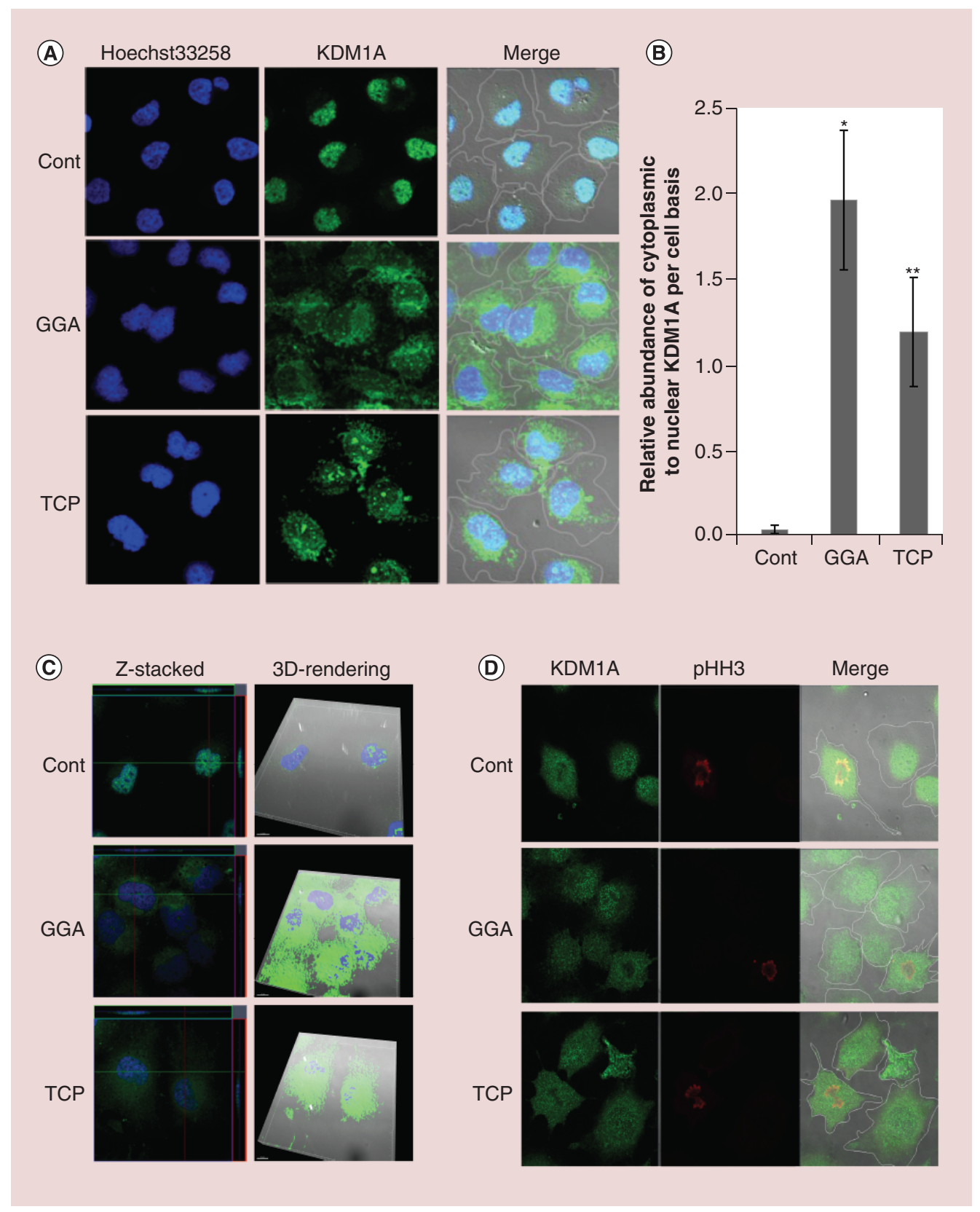

Figure 1. Cytoplasmic translocation of nuclear KDM1A induced by either GGA or TCP treatment. HuH-7 cells were incubated for $3 \mathrm{~h}$ with geranylgeranoic acid $(20 \mu \mathrm{M})$, trans-2-phenylcyclopropylamine $(5 \mu \mathrm{M})$ or vehicle alone as a control. (A) Images were obtained of immunofluorescence staining with anti-KDM1A primary antibody and anti-rabbit IgG-Alexa488 secondary antibody (green); nuclei were counter-stained with Hoechst 33258 (blue). Images of Hoechst 33258 and KDM1A were superimposed on the corresponding differential interference contrast images (Merge). (B) Quantitative analysis was performed on a cellular basis with the green fluorescent images in panel $A$ and relative abundance of the cytoplasmic to the nuclear fluorescence intensity was calculated. (C) In the merged Z-stacked images of KDM1A (green) with nucleus (blue), orthogonal hairlines show the XZ (green line and upper green box) and $Y Z$ (pink line and right pink box) planes in each panel. Z-stacked images were processed to obtain 3D renderings using Imaris software. The solid view of the 3D renderings allows confirmation of the cytoplasmic export of KDM1A (green) from the nucleus (blue) in GGA- and TCP-treated cells, whereas in control cells the majority of KDM1A is located in the nucleus. ${ }^{*} \mathrm{p}<0.05 ;{ }^{* *} \mathrm{p}<0.01$ (compared with control).

DIC: Differential interference contrast; GGA: Geranylgeranoic acid; TCP: Trans-2-phenylcyclopropylamine. 


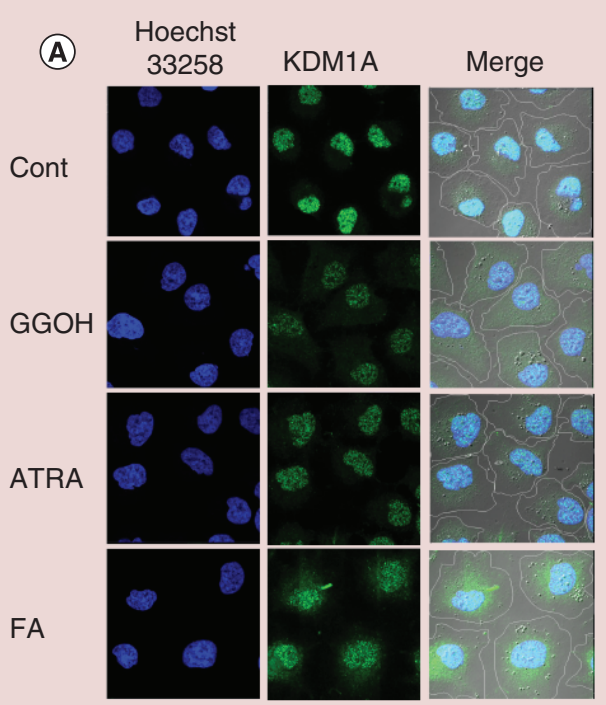

(B)

Hoechst 33258
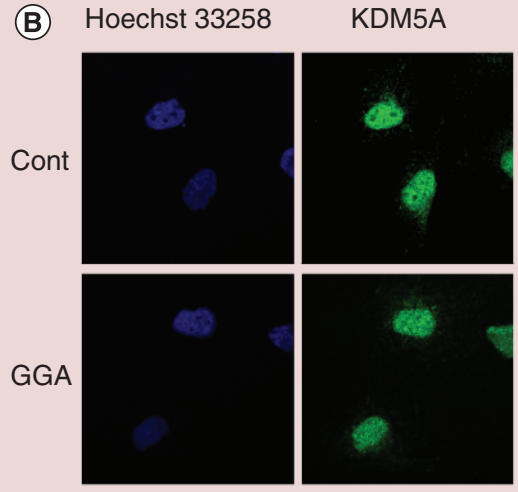

TCP
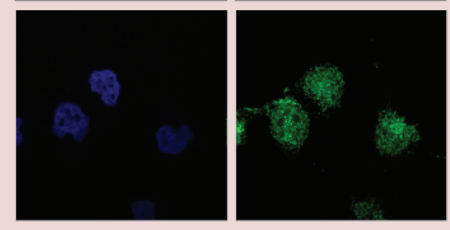

Merge
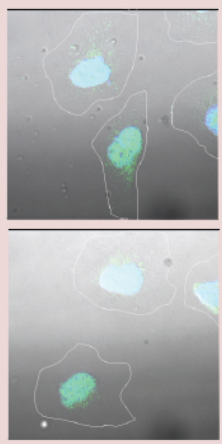

6.

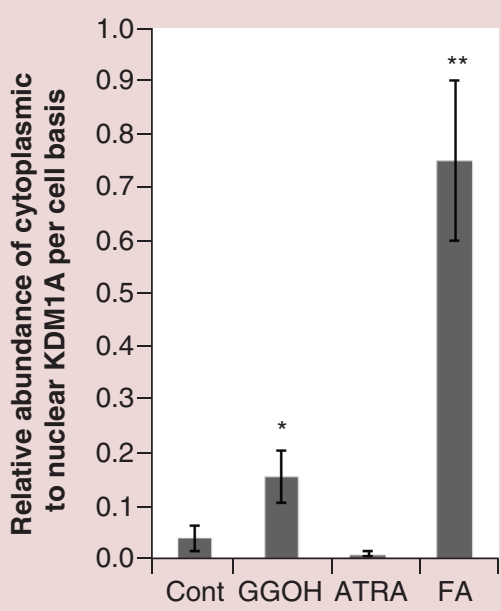

(C)
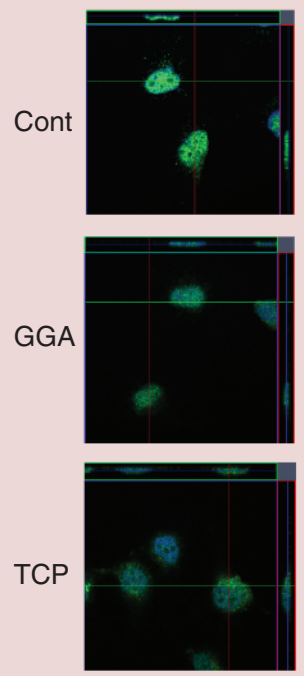

Figure 2. Nuclear localization of KDM1A and KDM5A after treatment with noninhibitory isoprenoids and KDM1A inhibitors for $3 \mathrm{~h}$.

(A) Immunofluorescence staining of KDM1A (green) and counter staining with Hoechst 33258 (blue) in HuH-7 cells treated for $3 \mathrm{~h}$ with the GGA-analogous isoprenoids geranylgeraniol, ATRA and FA. Each isoprenoid was used at a final concentration of $20 \mu \mathrm{M}$. Quantitative analysis was performed to calculate the relative fluorescence intensity. (B) After GGA or TCP treatment, cells were immunostained for KDM5A (green), another lysine-specific demethylase, and the nuclei were counterstained with Hoechst 33258 (blue). (C) In the merged images of KDM5A (green) and nucleus (blue) staining, orthogonal hairlines show the $X Z$ (green line and upper green box) and $Y Z$ (pink line and right pink box) planes in each panel.

${ }^{*} \mathrm{p}<0.05 ; * * \mathrm{p}<0.01$ (compared with control).

ATRA: All-trans retinoic acid; FA: Farnesoic acid; GGA: Geranylgeranoic acid; GGOH: Geranylgeraniol; TCP:

Trans-2-phenylcyclopropylamine.

Release of nuclear KDM1A by GGA or TCP under cell-free conditions

We recently reported that GGA directly inhibits recombinant human KDM1A [15]. TCP acts as an irreversible inhibitor forming a covalent adduct with the FAD (flavin adenine dinucleotide) cofactor of the KDM1A enzyme [26]. 

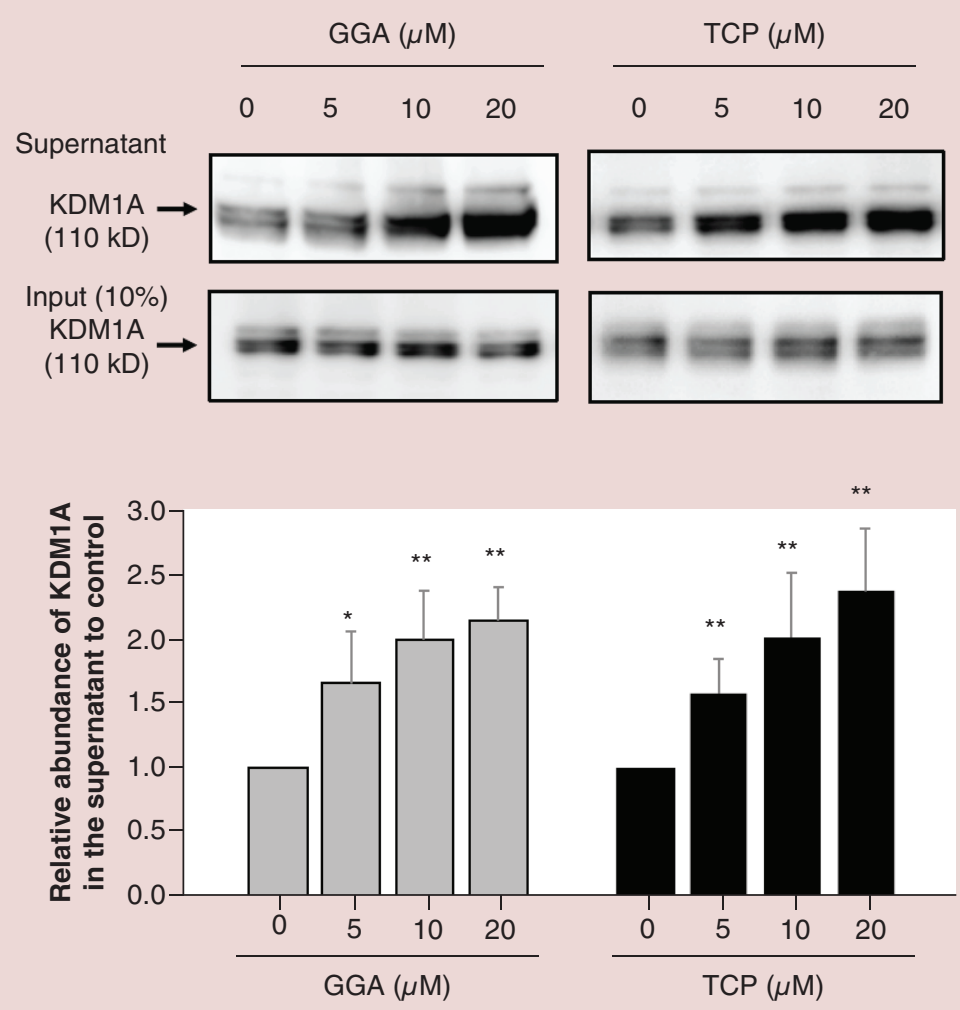

Figure 3. Release of nuclear KDM1A after incubation of the nuclear fraction with GGA or TCP (cell-free experiment). GGA and TCP directly released KDM1A from the nuclear fraction in a concentration-dependent manner. Nuclear fractions were treated with either GGA $(5-20 \mu \mathrm{M})$ or TCP $(5-20 \mu \mathrm{M})$ at $4{ }^{\circ} \mathrm{C}$ for $24 \mathrm{~h}$. After centrifugation, the supernatant was analyzed by SDS-PAGE and western blotting. The lower bar graph shows densitometric analysis of the KDM1A bands in the supernatant on the blots $(n=3)$.

${ }^{*} \mathrm{p}<0.05 ; * * \mathrm{p}<0.01$ (compared with $0-\mu \mathrm{M}$ control).

GGA: Geranylgeranoic acid; KDM1A: Lysine-specific demethylase 1A; TCP: Trans-2-phenylcyclopropylamine.

Here, we examined the direct effect of these inhibitors on the release of KDM1A protein from the nucleus. Dosedependent release of the protein from the nuclear fraction was observed with either GGA or TCP (Figure 3).

Finally, we investigated GGA-induced changes in the cellular level of KDM1A protein to understand why KDM1A translocates out of the nucleus upon GGA treatment. Cellular levels of KDM1A decreased in a timedependent manner after GGA treatment (Figure 4A). This suggests that GGA may induce KDM1A degradation by cytoplasmic translocation, because KDM1A transcript levels did not change during GGA treatment (Figure 4B).

\section{Discussion}

The present results demonstrate, for the first time, that GGA and TCP, small chemical inhibitors of KDM1A, induce cytoplasmic translocation of KDM1A protein from the nucleus in human hepatoma-derived cells. KDM1A is a component of the CoREST corepressor complex [27] and is a recognized nuclear marker protein [28]. Therefore, our finding of KDM1A inhibitor-induced translocation of KDM1A from the nucleus to the cytoplasm may be important for both inhibitor screening and understanding KDM1A biology. We discuss the biological and clinical significance of the transfer of KDM1A protein from the nucleus to the cytoplasm in response to KDM1A inhibitors.

We confirmed the strict nuclear localization of KDM1A protein in human hepatoma-derived HuH-7 cells before the drug treatment (Figure 1). KDM1A was discovered in 2004 as a nuclear homolog of amine oxidases that functions as a histone demethylase and transcriptional corepressor [3]. These functions indicate that the enzyme resides in chromatin and plays a role in the repression of gene expression through epigenetic mechanisms. Here, we describe the striking finding that treatment with the KDM1A inhibitors GGA and TCP caused the immediate translocation of KDM1A from the nucleus to the cytoplasm. This suggests that these inhibitors not only inhibit 

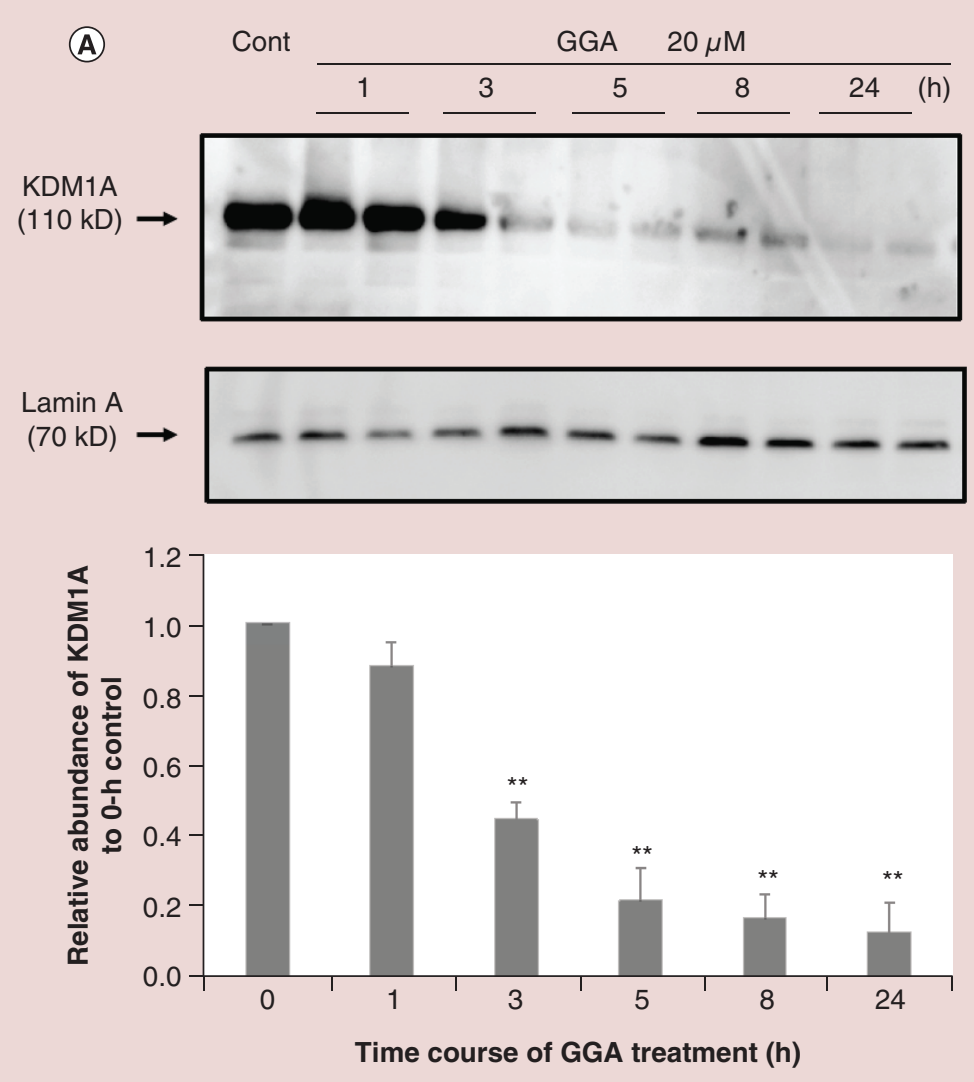

(B)

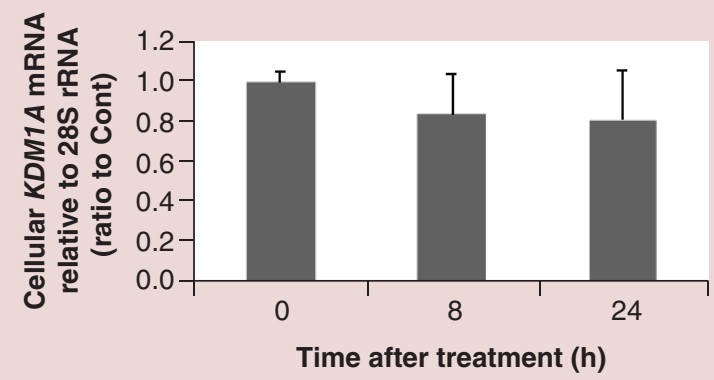

Figure 4. Downregulation of cellular KDM1A by GGA treatment (cellular experiment).

(A) Western blot of KDM1A protein in whole cell lysates of HuH-7 cells treated with $20 \mu \mathrm{M} \mathrm{GGA}$ for the indicated times. A lower panel of the blot shows loading controls stained with anti-lamin A antibody. The bar graph shows densitometric analysis of the KDM1A bands on the blots $(n=3)$. (B) KDM1A mRNA levels were analyzed by real time-qPCR after treatment of HuH-7 cells with $20 \mu \mathrm{M}$ geranylgeranoic acid for 8 or $24 \mathrm{~h}$.

${ }^{*} \mathrm{p}<0.05 ;{ }^{* *} \mathrm{p}<0.01$ (compared with 0 -h control).

GGA: Geranylgeranoic acid; TCP: Trans-2-phenylcyclopropylamine.

enzyme activity, but moved the enzyme itself to a location where epigenetic regulation was not possible. Therefore, these two inhibitors block the function of the enzyme via a dual mechanism.

We wanted to see if other compounds could induce the transfer of the KDM1A from the nucleus to the cytoplasm. We tested the ability of the GGA-analogous compounds GGOH (an alcoholic derivative of GGA), ATRA (a monocyclic and conjugated double bond derivative of GGA) and FA (an acyclic sesquiterpenoid acid [GGA is an acyclic diterpenoid acid]) to induce the cytoplasmic translocation of nuclear KDM1A. ATRA, which exhibits no KDM1A-inhibitory activity, did not induce KDM1A translocation. Both GGOH and FA, with less 
KDM1A inhibitory activity than GGA [15], slightly but significantly translocated KDM1A (Figure 2). Taken together, these results suggest that the activity of the inhibitors may be associated with the dislodging/translocating activity. Hence, an assay for drug-induced translocation of nuclear KDM1A could be developed to screen for KDM1A inhibitors without the need for measurement of enzyme activity.

A second question is whether these enzyme inhibitors dislodge only KDM1A from the chromatin. We investigated another lysine-specific demethylase, KDM5A, which plays a cooperative role in demethylation of histone $\mathrm{H} 3$ lysine 4 in conjunction with KDM1A [29]. We confirmed that KDM5A localized to the nucleus. Under the same conditions that dislodged nuclear KDM1A, neither GGA nor TCP induced translocation of nuclear KDM5A to the cytoplasm. This indicates that the inhibitors' effect on subcellular localization is specific for KDM1A. However, it remains unclear whether other components, such as HDAC1/2, may be released from the CoREST complex by GGA or TCP treatment.

Recruitment of KDM1A to chromatin has been extensively and thoroughly explored [30-34], but how KDM1A is unloaded from chromatin has received little attention. Nair et al., described the cell-cycle-dependent association and dissociation of KDM1A with chromatin [25]. KDM1A is recruited to the chromatin of cells in $G_{1} / S / G_{2}$ phases and is displaced from the chromatin of M-phase cells. We confirmed that KDM1A protein was distributed throughout the cell in M-phase cells stained with the pHH3 antibody (Figure 1D), and that in other non-M-phase cells KDM1A protein remained in the nucleus (Figure 1). However, in cells treated with GGA or TCP, KDM1A was distributed throughout the whole cell interior as in control M-phase cell, irrespective of cell-cycle stage. Therefore, GGA and TCP did not increase the number of cells in M-phase, but did act on cells in $G_{1} / S / G_{2}$ phases to induce the translocation of nuclear KDM1A to the cytoplasm. Anti-pHH3 antibody recognizes phosphorylated Ser10 on histone $\mathrm{H} 3$ and introduction of a negatively charged phosphate group on Ser10 completely abolishes KDM1A activity [35]. Taken together with the results presented here, one can reasonably speculate that a KDM1A inhibitor may unload the enzyme from chromatin as M-phase cells release KDM1A from chromatin.

Last, we speculate that direct binding of GGA or TCP to nuclear KDM1A might cause the release of the enzyme from the chromatin. Analysis of the nuclear fraction containing KDM1A, revealed that GGA- and TCPmediated release of KDM1A from the nucleus to the supernatant was dose dependent (Figure 3). Both GGA [15] and TCP [26] directly inhibit recombinant KDM1A enzyme activity and are thought to bind to the substrate binding site of the enzyme. The structures of free and CoREST-bound KDM1A are virtually identical, with only a small difference in the orientations of two $\alpha$-helices of the tower domain relative to the amine oxidase domain of KDM1A [36]. In this context, it is difficult to speculate whether direct binding of GGA or TCP to the substrate binding site in the KDM1A amine oxidase domain would affect interaction between the enzyme and the CoREST protein. Other inhibitor-induced conformational changes of KDM1A may be involved in its unloading from the nuclear fraction in the cell-free experiment. We should also consider the metabolic fate of the KDM1A exported in response to treatment with its inhibitor. GGA-induced downregulation of cellular KDM1A levels is time dependent (Figure 4). This may be explained by polyubiquitination and efficient degradation of the dislodged KDM1A from the chromatin by the proteasome system in the cytoplasmic space [37].

\section{Conclusion}

The present study clearly demonstrates that the GGA and TCP KDM1A inhibitors induce cytoplasmic translocation of nuclear KDM1A in human hepatoma cells. This biological process has the potential to be useful for screening promising cancer-preventive epigenetic therapeutic agents targeting KDM1A, and further work exploring this application is warranted.

\section{Future perspective}

As described in Introduction section, the 4,5-didehydro derivative of GGA (peretinoin or acyclic retinoid) potentially prevents second primary hepatoma in placebo-controlled, randomized, Phase II-III clinical trials [21-24]. Furthermore, the challenges of systemic treatment of solid tumors, such as hepatoma, with several VEGFR inhibitors (or orally active multitarget kinase inhibitors) and immune checkpoint inhibitors were often reported [38,39] and recently, were more intensively reviewed $[40,41]$. Some of them have been shown to be effective in ongoing Phase III randomized, placebo-controlled, clinical trials. We believe our present results will be of significant interest not only to cell biologists in general, but also to researchers working in the fields of developing preventive and therapeutic agents against clinical cancers, in particular, those studying hepatoma. Given the current clinical application of TCP as an antidepressant and its ongoing clinical trials on acute myeloid leukemia with ATRA [13], 
and that GGA is present in medicinal herbs [14], we need to continue our research until we show that either TCP or GGA should be clinically applied especially for complementary treatment and prevention of second primary hepatocellular carcinoma.

\section{Summary points}

- Geranylgeranoic acid (GGA) is a natural diterpenoid acid found in some medicinal herbs.

- GGA and its didehydro derivative are known to prevent experimental and clinical hepatocellular carcinogenesis.

- GGA and tranylcypromine (trans-2-phenylcyclopropylamine; TCP) inhibit histone-modifiable lysine-specific demethylase-1 (LSD1/KDM1A), an enzyme involved in epigenetic modification, which is localized to the chromatin and its expression is often up-regulated in hepatocellular carcinoma cells.

- Here we show that GGA and TCP induced cytoplasmic translocation of nuclear KDM1A in a human hepatoma-derived cell line.

- GGA did not affect subcellular localization of another epigenetic enzyme, histone lysine-specific demethylase, KDM5A, which is also localized to the chromatin and plays a role in histone lysine demethylation and co-operates with KDM1A.

- Among histone lysine-specific demethylases, GGA-induced translocation is specific for KDM1A.

- GGA and TCP are both potent and hopeful agents for complementary treatment and prevention of hepatocellular carcinoma.

\section{Author contributions}

Y Shidoji conceived and designed the experiments; S Yabuta performed the experiments; Y Shidoji and S Yabuta analyzed the data; and Y Shidoji and S Yabuta wrote the paper.

\section{Acknowledgements}

English writing support was provided by R Porter, from Edanz group (www.edanzediting.com/ac) for the editing of a draft of this manuscript funded from the University of Nagasaki.

Financial \& competing interests disclosure

This work was supported in part by a grant-in-aid from the Japan Society for the Promotion of Science (grant number 16K00862) and a research grant $B$ from the University of Nagasaki.

The authors have no other relevant affiliations or financial involvement with any organization or entity with a financial interest in or financial conflict with the subject matter or materials discussed in the manuscript apart from those disclosed.

\section{Open access}

This work is licensed under the Attribution-NonCommercial-NoDerivatives 4.0 Unported License. To view a copy of this license, visit http://creativecommons.org/licenses/by-nc-nd/4.0/

\section{References}

Papers of special note have been highlighted as: $\bullet$ of interest; $\bullet \bullet$ of considerable interest.

1. Buschbeck M, Hake SB. Variants of core histones and their roles in cell fate decisions, development and cancer. Nat. Rev. Mol. Cell Biol. 18, 293-314 (2017).

2. Feng Z, Yao Y, Zhou C et al. Pharmacological inhibition of LSD1 for the treatment of MLL-rearranged leukemia. J. Hematol. Oncol. 9 , 24 (2016).

3. Shi Y, Lan F, Matson C et al. Histone demethylation mediated by the nuclear amine oxidase homolog LSD1. Cell 119, 941-953 (2004).

- A discovery paper of KDM1A.

4. Schenk T, Chen WC, Göllner S et al. Inhibition of the LSD1 (KDM1A) demethylase reactivates the all-trans-retinoic acid differentiation pathway in acute myeloid leukemia. Nat. Med. 18, 605-612 (2012).

5. Sakamoto A, Hino S, Nagaoka K et al. Lysine demethylase LSD1 coordinates glycolytic and mitochondrial metabolism in hepatocellular carcinoma cells. Cancer Res. 75, 1445-1456 (2015).

-• Describes clinical significances of KDM1A activity in hepatocellular carcinoma.

6. Zhao ZK, Yu HF, Wang DR et al. Overexpression of lysine specific demethylase 1 predicts worse prognosis in primary hepatocellular carcinoma patients. World J. Gastroenterol. 18, 6651-6656 (2012). 
-• Describes clinical significances of KDM1A in hepatocellular carcinoma patients.

7. Duteil D, Tosic M, Lausecker F et al. LSD1 ablation triggers metabolic reprogramming of brown adipose tissue. Cell Rep. 17, 1008-1021 (2016).

8. Shen E, Shulha H, Weng Z, Akbarian S. Regulation of histone H3K4 methylation in brain development and disease. Philos. Trans. R. Soc. B Biol. Sci. 369(1652) 20130514 (2014).

9. Huang C, Cheng J, Bawa-Khalfe T, Yao X, Chin YE, Yeh ETH. SUMOylated ORC2 recruits a histone demethylase to regulate centromeric histone modification and genomic stability. Cell Rep. 15, 147-157 (2016).

10. Kashuba V, Protopopov A, Podowski R et al. Isolation and chromosomal localization of a new human retinoblastoma binding protein 2 homologue 1a (RBBP2H1A). Eur. J. Hum. Genet. 8, 407-413 (2000).

11. Jin Y, Kim TY, Kim MS, Kim MA, Park SH, Jang YK. Nuclear import of human histone lysine-specific demethylase LSD1. J. Biochem. 156, 305-313 (2014).

12. Maes T, Carceller E, Salas J, Ortega A, Buesa C. Advances in the development of histone lysine demethylase inhibitors. Curr. Opin. Pharmacol. 23, 52-60 (2015).

13. ClinicalTrials.gov A service of the U.S. National Institutes of Health. (2017). https://clinicaltrials.gov/ct2/show/NCT02261779; https://clinicaltrials.gov/ct2/show/NCT02717884; https://clinicaltrials.gov/ct2/show/NCT0227

- Clinical trials with trans-2-phenylcyclopropylamine have been in progress for acute myeloid leukemia patients.

14. Shidoji Y, Ogawa H. Natural occurrence of cancer-preventive geranylgeranoic acid in medicinal herbs. J. Lipid Res. 45, 1092-1103 (2004).

-• Describes natural occurrence of hepatoma-preventive geranylgeranoic acid (GGA).

15. Sakane C, Okitsu T, Wada A, Sagami H, Shidoji Y. Inhibition of lysine-specific demethylase 1 by the acyclic diterpenoid geranylgeranoic acid and its derivatives. Biochem. Biophys. Res. Commun. 444, 24-29 (2014).

-. Describes a discovery of GGA's inhibitory effect on KDM1A activity.

16. Sakane C, Ohta H, Shidoji Y. Measurement of lysine-specific demethylase-1 activity in the nuclear extracts by flow-injection based time-of-flight mass spectrometry. J. Clin. Biochem. Nutr. 56, 123-131 (2015).

17. Nakamura N, Shidoji Y, Yamada Y, Hatakeyama H, Moriwaki H, Muto Y. Induction of apoptosis by acyclic retinoid in the human hepatoma-derived cell line, HuH-7. Biochem. Biophys. Res. Commun. 207, 382-388 (1995).

18. Okamoto K, Sakimoto Y, Imai K, Senoo H, Shidoji Y. Induction of an incomplete autophagic response by cancer-preventive geranylgeranoic acid (GGA) in a human hepatoma-derived cell line. Biochem. J. 440, 63-71 (2011).

19. Iwao C, Shidoji Y. Induction of nuclear translocation of mutant cytoplasmic $\mathrm{p} 53$ by geranylgeranoic acid in a human hepatoma cell line. Sci. Rep. 4, 4419 (2014).

20. Iwao C, Shidoji Y. Polyunsaturated branched-chain fatty acid geranylgeranoic acid induces unfolded protein response in human hepatoma cells. PLoS ONE 10, e0132761 (2015).

21. Okita K, Izumi N, Ikeda $\mathrm{K}$ et al. Survey of survival among patients with hepatitis $\mathrm{C}$ virus-related hepatocellular carcinoma treated with peretinoin, an acyclic retinoid, after the completion of a randomized, placebo-controlled trial. J. Gastroenterol. 50, 667-674 (2015).

-• Placebo-controlled randomized clinical trial with 4,5-didehydro GGA for prevention of second primary hepatocellular carcinoma.

22. Okita K, Izumi N, Matsuji O et al. Peretinoin after curative therapy of hepatitis C-related hepatocellular carcinoma: a randomized double-blind placebo-controlled study. J. Gastroenterol. 50, 191-202 (2014).

23. Muto Y, Moriwaki H, Saito A. Prevention of second primary tumors by an acyclic retinoid in patients with hepatocellular carcinoma. $N$. Engl. J. Med. 340, 1046-1047 (1999).

24. Muto Y, Moriwaki H, Ninomiya M et al. Prevention of second primary tumors by an acyclic retinoid, polyprenoic acid, in patients with hepatocellular carcinoma. Hepatoma Prevention Study Group. N. Engl. J. Med. 334, 1561-1567 (1996).

-. Placebo-controlled randomized clinical trial with 4,5-didehydro GGA for prevention of second primary hepatocellular carcinoma.

25. Nair VD, Ge Y, Balasubramaniyan N et al. Involvement of histone demethylase LSD1 in short-time-scale gene expression changes during cell cycle progression in embryonic stem cells. Mol. Cell. Biol. 32, 4861-4876 (2012).

26. Hayward D, Cole PA. LSD1 histone demethylase assays and inhibition. Methods Enzymol. 573, 261-278 (2016).

27. Meier K, Brehm A. Chromatin regulation: how complex does it get? Epigenetics 9, 1485-1495 (2014),

28. Lai X. A reproducible method to enrich membrane proteins with high purity and high-yield for an LC-MS/MS approach in quantitative membrane proteomics. Electrophoresis 34, 809-817 (2013).

29. Vallianatos CN, Iwase S. Disrupted intricacy of histone H3K4 methylation in neurodevelopmental disorders. Epigenomics 7, 503-519 (2015). 
30. Sun GQ, Alzayady K, Stewart R et al. Histone demethylase LSD1 regulates neural stem cell proliferation. Mol. Cell. Biol. 30, 1997-2005 (2010).

31. Scionti I, Hayashi S, Mouradian S et al. LSD1 controls timely MyoD expression via MyoD core enhancer transcription. Cell Rep. 18, 1996-2006 (2017).

32. Kuppuswamy M, Vijayalingam S, Zhao LJ et al. Role of the PLDLS-binding cleft region of CtBP1 in recruitment of core and auxiliary components of the corepressor complex. Mol. Cell. Biol. 28, 269-281 (2008).

33. Gamble MJ, Kraus WL. Visualizing the histone code on LSD1. Cell 128, 433-434 (2007).

34. Cai C, He HH, Chen S et al. Androgen receptor gene expression in prostate cancer is directly suppressed by the androgen receptor through recruitment of lysine-specific demethylase 1. Cancer Cell 20, 457-471 (2011).

35. Forneris F, Binda C, Vanoni MA, Battaglioli E, Mattevi A. Human histone demethylase LSD1 reads the histone code. J. Biol. Chem. 280, 41360-41365 (2005).

36. Yang M, Gocke CB, Luo X et al. Structural basis for CoREST-dependent demethylation of nucleosomes by the human LSD1 histone demethylase. Mol. Cell 23, 377-387 (2006).

37. Piao L, Suzuki T, Dohmae N, Nakamura Y, Hamamoto R. SUV39H2 methylates and stabilizes LSD1 by inhibiting polyubiquitination in human cancer cells. Oncotarget 6, 16939-16950 (2015).

38. Tovoli F, Renzulli M, Granito A, Golfieri R, Bolondi L. Radiologic criteria of response to systemic treatments for hepatocellular carcinoma. Hepatic Oncol. 4, 129-137 (2017).

39. Kudo M. Systemic therapy for hepatocellular carcinoma: 2017 update. Oncology 93, 135-146 (2017).

40. Tovoli F, Negrini G, Benevento F, Faggiano C, Granito A. Systemic treatments for hepatocellular carcinoma: challenges and future perspectives. Hepatic Oncol. 5, HEP01 (2018).

41. Mody K, Abou-Alfa GK. Systemic therapy for advanced hepatocellular carcinoma in an evolving landscape. Curr. Treat. Options Oncol. 20(2), 3 (2019). 
